\title{
Auction Based Virtual Machine Live Migration Management
}

\author{
Wang $\mathrm{Xu}{ }^{1,2}$, Ni Hong ${ }^{1}$ and Guo Zhichuan ${ }^{1+}$ \\ ${ }^{1}$ National Network New Media Engineering Research Center, Institute of Acoustics, Chinese Academy of \\ Sciences \\ ${ }^{2}$ University of Chinese Academy of Sciences
}

\begin{abstract}
In cloud computing, resources of datacenters are, instead of bought, rented as virtual machines (VMs). Typically, a cloud datacenter is consisted of hosts with different performance specifications. Assigning virtual machines on suitable hosts will greatly improve the overall performance as well as reduce the operating cost of a datacenter. This work proposes a set of heuristics for VM live migration and an auction based algorithm to solve VM management problems in datacenters. VM live migration technique enables a service to transfer from one host to another within imperceptible down-time, which minimize the performance impact to the service quality. By introducing market based auction process into resource management algorithm to guarantee fitness between VMs and resources. Experiments show auction based algorithm proposed in this work is efficient in allocating and managing VMs resources, minimizing number of migrations, and increasing the overall performance.
\end{abstract}

Keywords: cloud resource allocation, load management, double auction, distributed system

\section{Introduction}

Cloud computing is becoming a trend technology in recent years [1], [2], [3]. By renting instead of buying resources, cloud computing enables the users to extend or trim the computing power according to current demand flexibly. In cloud computing, a cloud datacenter provides virtualized computing service with a network of heterogeneous commodity servers. Resources of a datacenter is accessed through allocated virtual machines (VMs), e.g., realized by Docker, which are allocated at the user's specific requests. In general, both datacenters and user requirements are heterogeneous [4]. As in [5], cloud datacenters use VM live migration to accomplish dynamic load management. VM live migration is a technique enables VMs to migrate from a host to another without notable interruptions [6].

An auction is a process of buying and selling goods or services that has no standard value. Continuous double auction (CDA) [7] is a market based resource management and is becoming popular recently [8], [9], [10] And it has been proved that market based methods are quite effective in resource allocation problems in cloud environment [9], [10]. By employing the supply and demand mechanism with the auction method, the so called invisible hand of market will take it over to balance resource allocation and management problem within a datacenter. The researchers present a resource management algorithm named Auction based Cloud Resource Management Algorithm (ACRMA). It's a CDA based algorithm aiming to solve resource allocation and management problems within a datacenter using market theories.

In this paper, the researchers present a comprehensive architecture for cloud datacenters resource management via market based double auction. The rest of this paper is organized as follows: Section 2 provides detailed discussion of the host-wide heuristics. Section 3 shows auction details in cloud market. Section 4 includes experimental evaluations. Section 5 presents some conclusions and some important future directions.

\footnotetext{
+ Corresponding author. Tel.: + 8618612062648; fax: +861082547890.

E-mail address: guozc@dsp.ac.cn
} 


\section{VM Selection Heuristics}

\subsection{Host selection heuristics}

Host selection heuristics are used to select overloaded hosts who may want to migrate some of its VMs to other hosts. By trade off decision, only CPU and RAM are taken into observation.

A load management policy based on sliding time window is employed. Taking the CPU utilization ratio, given that a sliding time window length is $T$, at one certain time $t_{0}$, average CPU utilization ratio in time window is

$$
H_{i} \cdot U_{C P U}^{T}(t)=\sum_{t=t_{0}-T}^{t_{0}} H_{i} \cdot U_{C P U}(t)
$$

In which $H_{i} \cdot U_{C P U}(t)$ stands for the observed CPU utilization ratio of host $i$ at time $t$.

Given that utilization ratio thresholds of CPU and RAM on a certain host $i$ are $H_{i} \cdot U_{C P U}^{T H}$ and $H_{i} \cdot U_{R A M}^{T H}$ respectively. On conditions where $H_{i} \cdot U_{C P U}^{T}(t) \geq H_{i} \cdot U_{C P U}^{T H}(t)$ or $H_{i} \cdot U_{R A M}^{T}(t) \geq H_{i} \cdot U_{R A M}^{T H}(t)$, host is experiencing relatively high CPU load or memory shortage or both. Each host may not have the same threshold

\subsection{VM Selection Heuristics}

VM selection heuristics are used for selecting the VMs to be migrated. A host select one or more VM for migration once overloaded. Expecting to quickly decrease load, selection of the VM with the greatest resource consumption is preferred. For a VM, its overall utilization ration can be expressed as follows

$$
H_{i} \cdot U_{A L}^{V M}=\lambda_{C P U} H_{i} \cdot U_{C P U}^{V M}+\lambda_{R A M} H_{i} \cdot U_{R A M}^{V M}
$$

In which $\lambda_{C P U}$ and $\lambda_{R A M}$ are weight factors of CPU and RAM load respectively, where $\lambda_{C P U}+\lambda_{R A M}=1$ and $\lambda_{C P U}, \lambda_{R A M} \in[0,1] . \lambda_{C P U}$ and $\lambda_{R A M}$ can be further expressed as follows to reflect the CPU and RAM loads

$$
\lambda_{C P U}=\frac{H_{i} \cdot U_{C P U}^{V M}}{H_{i} \cdot U_{C P U}^{V M}+H_{i} \cdot U_{R A M}^{V M}}, \lambda_{R A M}=\frac{H_{i} \cdot U_{R A M}^{V M}}{H_{i} \cdot U_{C P U}^{V M}+H_{i} \cdot U_{R A M}^{V M}}
$$

Then

$$
H_{i} \cdot U_{A L}^{V M}=\frac{\left(H_{i} \cdot U_{C P U}^{V M}\right)^{2}+\left(H_{i} \cdot U_{R M}^{V M}\right)^{2}}{H_{i} \cdot U_{C P U}^{V M}+H_{i} \cdot U_{R A M}^{V M}}
$$

In this way, $H_{i} \cdot U_{A L}^{V M}$ pays more attention to the aspect with higher value. Finally, VMs with higher $H_{i} \cdot U_{A L}^{V M}$ values have higher priorities to be migrated.

\section{Auction in VM Allocation}

\subsection{Pricing tactics}

Multiple tactics are taken in to account to deal with different problems during auction. In this work, two tactics are taken, remaining time and resource.

Within a given bargaining period $\tau$, suppose that at a certain time $t$, the remaining time of a bargain is $\delta(t)$, so that $\delta(t) \in[0, \tau]$. Given resource $i$ 's minimum and maximum asking price for is $a_{i}^{\min }$ and $a_{i}^{\max }$ respectively. At time $t, a_{i}(t) \in\left[a_{i}^{\min }, a_{i}^{\max }\right] . a_{i}(t)$ starts at $a_{i}^{\max }$ and, as the time approach the deadline, decreases to $a_{i}^{\min }$ eventually. So $a_{i}(t)$ can be express as $a_{i}(t)=a_{i}^{\min }+f(\delta(t))\left(a_{i}^{\max }-a_{i}^{\min }\right)$. In which $f$ is a function of $\delta(t)$, which calculates the asking price. Choosing a proper expression for function $f$ can be miscellaneous. [12] gives out a mostly commonly used polynomial expression in their work

$$
f(x)=f^{0}+\left(1-f^{0}\right)\left(\frac{x}{x_{\max }}\right)^{\frac{1}{\beta}}
$$

It's easy to conclude that host $i$ 's asking price $a_{i}(t)$ and the bidding price $b_{i}(t)$ as follows

$$
\begin{aligned}
& a_{i}(t)=a_{i}^{\text {max }}-\left(a_{i}^{\max }-a_{i}^{\text {min }}\right)\left(\frac{\tau-\delta(t)}{\tau}\right)^{\frac{1}{\beta}} \\
& b_{i}(t)=b_{i}^{\text {min }}+\left(b_{i}^{\text {max }}-b_{i}^{\text {min }}\right)\left(\frac{\tau-\delta(t)}{\tau}\right)^{\frac{1}{\beta}}
\end{aligned}
$$


As for resources, the actual amount of resources is taken as price, thus prices are relatively fixed during bargaining period compare. However, by slightly increasing the asking price, the possibility of subsequent migrations can be reduced. Base asking price of a VM for CPU is $a_{C P U}=V M \cdot U_{C P U} \times V M \cdot M I P S \times V M \cdot P E$. And base asking price of a VM for RAM is $a_{R A M}=V M \cdot U_{R A M} \times V M \cdot R A M$.

Suppose the minimum and maximum asking prices of resource $k$ are $a_{k}^{\min }=\mu_{a}^{\min } a_{k}$ and $a_{k}^{\max }=\mu_{a}^{\max } a_{k}$, where $1<\mu_{a}^{\min } \leq \mu_{a}^{\max }$. In which $\mu_{a}^{\min }$ is set greater than 1 to ask for more resource to reduce the possibility of subsequent migrations. When $\mu_{a}^{\min }=\mu_{a}^{\max }$, asking price of resource $k$ stay constant within a bargain period. Similarly, base bidding price of host $i$ for CPU resource is $b_{C P U}=\left(H_{i} \cdot U_{C P U}^{T H}-H_{i} \cdot U_{C P U}\right) \times H_{i} \cdot M I P S \times H_{i} \cdot P E$. While base bidding price of host $i$ for RAM resource is $b_{C P U}=\left(H_{i} \cdot U_{R A M}^{T H}-H_{i} \cdot U_{R A M}\right) \times H_{i} \cdot R A M$.

Identical to the asking price, the minimum and maximum bidding prices of resource $k$ are $b_{k}^{\text {min }}=\mu_{b}^{\text {min }} b_{k}$ and $b_{k}^{\max }=\mu_{b}^{\max } b_{k}$, where $1<\mu_{b}^{\min } \leq \mu_{b}^{\max }$.

The overall ask and bidding prices for sellers and buyers will be the aggregation of above-mentioned tactics. Asking and bidding prices resource $r$ on host $i$ at time $t$ are

$$
\begin{aligned}
& H_{i} \cdot a_{r}(t)=a_{r}^{\text {max }}-\left(a_{r}^{\text {max }}-a_{r}^{\text {min }}\right)\left(\frac{\tau-\delta(t)}{\tau}\right)^{\frac{1}{\beta}} \\
& H_{i} \cdot b_{r}(t)=b_{r}^{\text {min }}+\left(b_{r}^{\text {max }}-b_{r}^{\text {min }}\right)\left(\frac{\tau-\delta(t)}{\tau}\right)^{\frac{1}{\beta}}
\end{aligned}
$$

\subsection{Trade mechanism}

Suppose the set of sellers is A and the set of buyers is B . For sellers, the set of sellers succeeded in auction for resource $r$ is

$$
\mathbf{A}_{\mathbf{r}}=\mathbf{A}-\left\{H_{x} \mid H_{x} \cdot a_{r}>H_{y} \cdot b_{r}, H_{x} \in \mathbf{A}, H_{y} \in \mathbf{B}\right\}
$$

In which $H_{x} \cdot a_{r}$ stands for the asking price for resource $r$ by seller $x$ and $H_{y} \cdot b_{r}$ stands for the bidding price for resource $r$ by buyer $y$. Sellers that succeeded in all auctions for each kind of resource are

$$
\mathbf{A}_{\text {quailify }}=\bigcap \mathbf{A}_{\mathbf{r}}
$$

Sort each $\mathbf{A}_{\mathbf{r}}$ in descending order. Suppose a seller $i$, where $H_{i} \in \mathbf{A}_{\mathbf{r}}$, ranks $\operatorname{rank}_{i}^{r}$ (the higher in ranking, the lower in value) in $\mathbf{A}_{\mathbf{r}}$, which has $n_{a r}$ members in total. The overall raking factor of seller $i$ in $\mathbf{A}_{\text {quailify }}$ is defined as follows

$$
\operatorname{rank}_{i}=\sum_{r} \operatorname{rank}_{i}^{r}+\frac{1}{2} \frac{\sum_{r}\left|\operatorname{rank}_{i}^{r}-\overline{\operatorname{rank}_{i}^{r}}\right|}{n_{a r}}+\frac{1}{4} \frac{\min \left(\operatorname{rank}_{i}^{r}\right)}{n_{a r}}
$$

Suppose all available buyer are $\mathbf{B}_{\text {available }}$ For a certain seller $i$, the available buyers whose bidding prices for each kind of resource are higher than the asking price of seller $i$ for the corresponding resource are

$$
\mathbf{B}_{\text {qualify }}^{\mathbf{i}}=\bigcap\left\{H_{x} \mid H_{i} \cdot a_{r} \leq H_{x} \cdot b_{r}, H_{x} \in \mathbf{B}\right\} \cap \mathbf{B}_{\text {available }}
$$

Sort $\mathbf{B}_{\text {qualify }}^{\mathbf{i}}$ in descending order similarly. Let sellers and buyers be paired as follows:

1. Take $H_{i}$ out of $\mathbf{A}_{\text {quailify }}$, where $H_{i}$ has the highest overall ranking in $\mathbf{A}_{\text {quailify }}$;

2. Take $H_{j}$ out of $\mathbf{B}_{\text {qualify }}^{\mathbf{i}}$, where $H_{j}$ has the highest overall ranking in $\mathbf{B}_{\text {qualify }}^{\mathbf{i}}$;

3. $H_{i}$ sells to $H_{j}$, then render $H_{i}$ and $H_{j}$ unavailable for subsequent steps,

5. Repeat 1 to 4 until $\mathbf{A}_{\text {quailify }}$ or $\mathbf{B}_{\text {available }}$ is empty.

\section{Experimental Evaluation}

\subsection{Evaluation method}

This experiment involved 18 hosts. Hosts were divided into 3 group. Hosts in the same group shared the same system configuration. Hosts in different groups had different combination of configuration set, simulating a heterogeneous datacenter. Host specifications are listed in Table 1. Overutilization thresholds were set to start migrations, while underutilization thresholds were set to stop hosts from bidding for VMs. 
Table 1: Specifications of Hosts

\begin{tabular}{|l|l|l|l|}
\hline & Group 1 & Group 2 & Group 3 \\
\hline Processing Element & 8 & 32 & 80 \\
\hline MIPS & 3500 & 2400 & 2000 \\
\hline Memory/GB & 128 & 256 & 512 \\
\hline CPU utilization threshold (Over/Under) & $0.5 / 0.4$ & $0.7 / 0.6$ & $0.9 / 0.8$ \\
\hline RAM utilization threshold (Over/Under) & $0.5 / 0.4$ & $0.7 / 0.6$ & $0.9 / 0.8$ \\
\hline
\end{tabular}

The management agent submitted VM allocation request. The arrival and departure of VMs were modelled as Poisson processes. Each VM was configured to be allocated with 1, 2, 4, 6, 8 virtual cores and 2, $4,8,12$, 16GB memory, simulating VM heterogeneity. Number of VMs with specific amount of resource of each kind fit normal distribution separately.

Random heuristics were used as a baseline in this experiment, as random heuristics are commonly used as a baseline to compare between different methods. When a host is overloaded, random heuristics select a random VM from this host and migrate the VM to a qualified random host, which is still not overloaded, being totally unaware of host-wide resources management details.

\subsection{Result \& discussion}

Fig. 1 shows the average CPU and RAM utilization level of each type of hosts respectively. ACRMA showed stronger ability to stabilize resource usage than benchmark algorithm. While trying to maintain higher resource utilization ratios, ACRMA did render some hosts overutilized a little. However, making better use of host resources enabled ACRAMA to host 815 VMs within simulation limit, while random algorithm hosted 693 VMs.
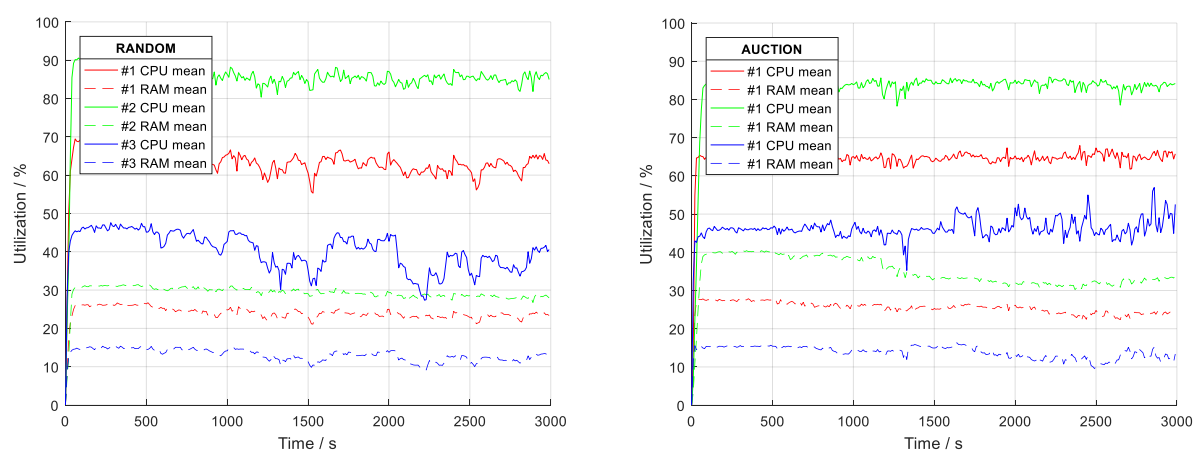

Fig. 1: Utilization statics grouped by host type

In the starting phase, ACRMA algorithm tried to keep fewer hosts active as well as to keep balanced host utilization ratios. During the auction process of ACRMA algorithm, the highest bidders, those who offer the most, win the bid. In this simulation, the group of hosts with the most available resources took turns to win the bid until the group was no longer the highest bidder. However, random heuristics selected host randomly when allocating new VMs, making each host selected in equal probabilities, thus number of active hosts raised up to maximum in a short time.

The imbalance of CPU and RAM utilization is because the natural imbalance of overall VM resource consumptions in statics, which is normal in realistic sceneries. Since we trade each kind of resource equally, resource imbalance in consumption will not compromise the effect of this algorithm. As the result implies, due to better fitness between VMs and hosts inherited from auction process, our algorithm is still effective to deal with VM allocations with imbalanced resource consumptions.

VM live migration mainly consists of two phases, VM selection and VM allocation or relocation. It should be noted that this study mainly focus on the probability of introducing market based methodology into live migration management in VM allocation phase In VM selection phase, we still utilized a series of traditional heuristics to identify VMs, which should be migrated.

In future works, decentralization of auction process will be an aspect that is worth investigating. As in current version of auction process, there has to be an auctioneer knowing some global information. However, 
as a cloud datacenter usually contains thousands or even more number of servers, the scalability of this algorithm will be limited by the auctioneer itself. ACRMA with modifications, utilizing a distributed auction algorithm as proposed in [13], may resolve this scalability problem.

\section{Conclusion}

In this work, the researchers present an auction based method to deal with the resource allocation and management problem in cloud environment. It has been proved to be effective in experimental simulations. By introducing auction based market methods, a cloud container with higher requirements is able to find a host with higher available resource capacity. Thus, heterogeneous loads are able to get their suitable resources, and at the same time, heterogeneous hosts are well balance according to their capabilities, which improves the overall efficiency. Fitness between cloud resource and virtual machines is guaranteed.

\section{Acknowledgements}

This work is partly supported by the National High Technology Research and Development Program of China (863 Program) (Project No. 2015AA015802). And the authors would like to thank the anonymous referees for their comments and suggestions.

\section{References}

[1] S. Srinivasan and V. Getov: 'Navigating the cloud computing landscape-technologies, services, and adopters', Computer, 2011, 44(3), 22-23.

[2] A. T. Velte, T. J. Velte, R. C. Elsenpeter, and R. C. Elsenpeter: 'Cloud computing: a practical approach', 2010, McGraw-Hill New York.

[3] L. Hongjia, C. Song, L. Tao, W. Heng, and W. Zejue: 'Research on Green Index Based Energy Consumption Modeling Method for Cloud Computing System', Journal of Network New Media, 2013(1), 38-42.

[4] M. A. Netto, C. Vecchiola, M. Kirley, C. A. Varela, and R. Buyya: 'Use of run time predictions for automatic coallocation of multi-cluster resources for iterative parallel applications', Journal of Parallel and Distributed Computing, 2011, 71(10), 1388-1399.

[5] C.-T. Fan, W.-J. Wang, and Y.-S. Chang: 'Agent-based service migration framework in hybrid cloud', High Performance Computing and Communications (HPCC), 2011 IEEE 13th International Conference on, 2011, IEEE, 887-892.

[6] W. Voorsluys, J. Broberg, S. Venugopal, and R. Buyya: 'Cost of virtual machine live migration in clouds: A performance evaluation', IEEE International Conference on Cloud Computing, 2009, Springer, 254-265.

[7] M. Posada, C. Hernandez, and A. Lopez-Paredes: 'Learning in continuous double auction market', in 'Artificial Economics', 41-51; 2006, Springer.

[8] Y. Lan, W. Tong, Z. Liu, and Y. Hou: 'Multi-unit continuous double auction based resource allocation method', Intelligent Control and Information Processing (ICICIP), 2012 Third International Conference on, 2012, IEEE, 773-777.

[9] P. Bonacquisto, G. Di Modica, G. Petralia, and O. Tomarchio: 'A strategy to optimize resource allocation in auction-based cloud markets', Services Computing (SCC), 2014 IEEE International Conference on, 2014, IEEE, 339-346.

[10] Y. Zhang, K. Xu, X. Shi, H. Wang, J. Liu, and Y. Wang: 'Continuous double auction for cloud market: Pricing and bidding analysis', Wireless Communications and Networking Conference (WCNC), 2016 IEEE, 2016, IEEE, 1-6.

[11] H. J. Curnow and B. A. Wichmann: 'A synthetic benchmark', The Computer Journal, 1976, 19(1), 43-49.

[12] P. Faratin, C. Sierra, and N. R. Jennings: 'Negotiation decision functions for autonomous agents', Robotics and Autonomous Systems, 1998, 24(3), 159-182.

[13] D. P. Bertsekas and D. A. Castañon: 'Parallel synchronous and asynchronous implementations of the auction algorithm', Parallel Computing, 1991, 17(6), 707-732. 